\title{
OBITUARIO: LA APASIONADA OBRA DE \\ Donald Guillermo Jackson Squella \\ Y SU MUERTE INESPERADA (1960-2015)
}

\author{
Lautaro Núñez \\ Instituto de Investigaciones Arqueológicas y Museo \\ Universidad Católica del Norte \\ San Pedro de Atacama
}

\begin{abstract}
"Cuando el sol de la muerte
se beba toda el agua de tus ríos

y sus rayos voraces

mortifiquen tu piel y la resequen

el agua de tu cuerpo ascenderá a los cielos

y convertida en sangre

lloverá una vez más sobre los cauces"

(Oscar Hahn, Meditaciones al atardecer

1996:168)
\end{abstract}

Donald se durmió lentamente junto a su muerte. Se acompañaron con la misma pasión que puso en cada una de sus acciones, atrás de su mirada y habla tan clara como penetrante y atrayente. Para quienes desde otras regiones revisen su obra descubrirán una creatividad y persistencia insospechadas en sus propias propuestas, que las siguió en escritos de menor a mayor complejidad, hasta alcanzar ribetes que tocaron grandes temas sudamericanos. Y que esta ausencia involuntaria y dolorosa haya ocurrido a sus escasos 54 años de edad, nos ha afectado en lo más profundo, porque había alcanzado una precisa armonía entre vida, ciencia y formación en los comienzos de su madurez total ¿Cómo se puede aceptar esto en un ser cuyo discurso palpitaba de tanta vitalidad y en el medio de su rebeldía natural? ¿Cómo se resistió al tironeo entre la esperanza de seguir, con la asistencia de los dioses Mayas, y el tránsito hacia el misterio definitivo? De verdad, lo mantendremos vivo en nuestras memorias en la medida que nosotros mismos seamos capaces de transferirlo a las nuevas generaciones, lejos del olvido y cerca de aquellos elegidos que nos dejaron esta disciplina más cierta, crecida y prestigiosa.

Donald vivía largas temporadas en Los Vilos donde sus padres tenían una casa de verano y por lo mismo, ése era su paraje vital donde ya desde niño lo había auscultado en todos sus rincones. El acercamiento a ese paisaje, donde lo continental y costero se friccionan en una atmosfera de habitabilidad estimulante, lo marcó a tal punto que nunca dejó de investigar su tierra "natal" de verdad, aunque naciera en Talca. Se formó allí como si fuera sujeto y objeto de su propia curiosidad.

Fue en el año 1974 cuando junto a su hermano gemelo Douglas corrían con un extraño atrapa mariposas junto al inicio de nuestras excavaciones en Quereo, precisamente junto a Los Vilos. Douglas siguió persiguiéndolas y hoy es un ferviente naturalista. Donald, en cambio, se detuvo y nos observó por una hora en absoluto silencio. De súbito escuchamos la pregunta que nos había atormentado durante el comienzo de esa excavación extendida: "¿Perdón, este es un sitio arqueológico o paleontológico?" Casamiquela (paleontólogo), Varela (geólogo) y yo mismo no podíamos creerlo. Nos miramos y le dije: "¿Quieres ser nuestro asistente?". Tenía sólo 14 años y nos saludamos como si fuera mayor, sin antes entregarle un borde erosionado de la excavación para que con espátula en mano hiciera lo que nos había visto hacer. En esa misma tarde este adolescente brochaba una vértebra de mastodonte del nivel superior de Quereo y su mirada profunda y seductora fue la misma que hoy recordamos, porque Donald tenía un extraño don de hablar con los ojos y fue así como se hizo parte diaria del equipo durante toda la excavación. Sólo volvía a dormir a 
su casa. Había captado plenamente nuestra propuesta de constituir, particularmente entre sitios tempranos, la aplicación de excavaciones extensas con decapado cepillado, plantas in situ, harneo de agua, flotación y muestreos interdisciplinarios. Tal orientación lo acompañó en toda su trayectoria científica.

Pasaron los años y llegó el momento de la Universidad. Estaba tan motivado por esta disciplina que en esos años de temprana juventud liceana ya era un gran activista de las Juventudes Científicas que Grete Mostny había congregado en torno al Museo de Historia Natural. Posteriormente, Donald optó por sus estudios de pregrado en México. Con este fin debí asistir a una reunión con sus padres para asegurarles que les escribiría a mis colegas en México para su recibimiento y orientación. Así ocurrió. Esto explica que siempre se asoció a los colegas con quienes habíamos iniciado los principios de la arqueología social latinoamericana y por ello nunca dejó de señalar que toda teoría social debería sustentarse en la praxis. Por cierto, fue parte sustancial del ideario de cambios sociopolíticos que aspiramos antes y después de la dictadura, ahora motivado por sus profesores de Ciudad de México. En consecuencia, se recibió de arqueólogo el año 1985 en la Escuela Nacional de Antropología e Historia de México.

De regreso, juntos, compartíamos totalmente nuestra devoción regionalista y percibíamos la ciencia con conciencia social, ejercida en su caso hacia la comunidad de Los Vilos que lo distinguió como un hijo pródigo. Por lo mismo, yo no sabía que allí habían levantado un monumento al mastodonte hasta que se me apareció en la oscuridad cuando volví largo tiempo después. Fue como una aparición desafiante de los restos excavados, ahora articulado, de aquel de Quereo. Por supuesto que estuvo su mano en torno a esta iniciativa y este recuerdo, cuando lo compartimos, de nuevo nos unía la idea de proyectar en el sitio Quereo un museo abierto donde se representen a escala real los megamamíferos registrados en el área de Los Vilos.

No dejó de escudriñar "su" pasado hasta revelarlo desde la localidad para comprender las conductas humanas a escalas mayores y tan amplias que sus últimos escritos alcanzaron impactos internacionales. Esta intensidad empírica y afecto por "su" Semiárido lo llevaron a valorar y evaluar la mayor cantidad y calidad de sitios en espacios restringidos. Así, sabía donde estarían los datos duros que durante su vida fueron de tal solvencia que sus juicios eran consistentes e indudables. Donald sabía bien lo que ha- bía ocurrido en la superficie del territorio, pero el mismo, con sus colaboradores excavaba con delicadeza para crear y manejar sus propias fuentes documentales, en un marco de alta convocatoria, sin sobredimensionar su notable maestría. Sabía escuchar, aceptar, corregir y respetar los aportes de cada cual, de tal modo que siempre tuvo como único protagonista a la sociedad del pasado. Estas virtudes las expresó fielmente en aquellos tiempos en que fue un líder genuino de la Sociedad Chilena de Arqueología.

Luego en el año 1993 se incorpora a su querida Universidad de Chile, donde ejerció hasta el final en el Departamento de Antropología de la Facultad de Ciencias Sociales, donde obtiene el grado de Magíster en Arqueología el año 2002. Su compromiso con su Universidad y por consiguiente con el carácter del quehacer académico lo llevó a comentarme sobre el peligro de la mercantilización del oficio y la necesidad de otorgarle a los alumnos una sólida base científico-técnica, de modo que cual sea la orientación de sus profesiones la visión académica debería guiar sus intervenciones.

En su calidad de Profesor Asociado venía construyendo últimamente una carrera académica ejemplar, plenamente identificado con los paisajes culturales del "Norte Semiárido", donde orientó sus líneas de acción en la problemática de caza-pesca y recolección. Tenía un particular interés por los poblamientos tempranos con especial énfasis en las ocupaciones costeras, siempre rodeado de estudiantes y colegas en el medio de su persistente orientación interdisciplinaria.

Ejerció docencia en Teoría Arqueológica I, Prehistoria de Chile I (Norte Chico), Métodos y Técnicas de Laboratorio: Lítica, y seminarios de especialización sobre sociedades cazadoras-recolectoras-pescadoras. Estas materias se reflejaron además en la conducción de más de diez tesis que cubrieron temas relevantes del Semiárido, con extensiones hacia el extremo sur del país, los bosques templados, el norte árido, incluyendo la costa desértica. Recuerdo otras tesis sobre tecnología lítica en la materialidad ignimbrítica por el norte, las prácticas de reducción bifacial, la lítica de Cobija o las evidencias Huentelauquén al interior de la costa.

Donald estableció una estricta relación entre su sonrisa y sus proyectos de investigación y docencia, explicándose de este modo el notable hecho de hacer ciencia en estado de alegría permanente. Surgía así su ferviente interés en 
la creación de conocimientos desde una praxis concreta y su transferencia directa ante sus alumnos, recomendando siempre evitar los excesos de interpretaciones. En las palabras de César Méndez: "Una docencia donde se valoró más la experiencia del hacer, que solamente los conocimientos de los textos". Ciertamente, acordábamos que los datos de "segunda mano", los cateos en grandes yacimientos, dataciones sin críticas internas, sumados a excesos especulativos, conducen a la impotencia científica y que estas publicaciones derivadas de interpretaciones, por más ingeniosas que sean, duran menos que una bolsa de "cabritas" en el medio de un espectáculo de citas bibliográficas de moda.

Efectivamente, Donald, un fiel cazador de datos propios, fue investigador principal en seis proyectos y coinvestigador en otros seis, en su mayoría provenientes de los concursos FONDECYT de donde derivaron un impresionante listado de publicaciones iniciadas por el año 1987. Fue primer autor de dos libros y coautor de otro. Escribió como primer autor cinco capítulos de libros y dos en calidad de coautor. En las revistas nacionales publicó como primer autor 36 artículos y 17 de coautor, que se acompañaron con diez contribuciones en revistas internacionales en su calidad de primer autor y ocho como coautor. Y en este recuento no podríamos dejar su activa e inolvidable participación en congresos y eventos nacionales e internacionales con centenas de ponencias como autor principal y coautor. Al hojear en general sus publicaciones es importante detectar con quien compartió sus autorías. De hecho, salvo error u omisión, en varias oportunidades lo hizo con su gran amigo, colega y compañero de ruta, César Méndez, quien lo acompañó y honró hasta el final. Precisamente, últimamente nos reunimos los tres con Calogero Santoro en el célebre "laboratorio del Donald" para armar eventos y futuras publicaciones. Además, escribió con Roxana Seguel, porque ambos se educaron mutuamente en sus materias y compartieron afectos, labor y vida. Sin Roxana a su lado, no habría incorporado las prácticas de conservación como un caso único en el país. Publicó con Mauricio Massone, Pedro Báez, Mónica Bahamondes, Alfredo Prieto y Patricio López. Por lo anterior, se desprende que buscaba en sus coautorías la presencia de expertos en otras miradas interdisciplinarias, que incluso implicó a su hermano Douglas, con sus aportes desde las ciencias naturales, básicamente en estudios de bivalvos y moluscos dulceacuícolas terrestres. Particularmente compartí algunas publicaciones sobre la industria lítica de Tambillo del desierto de Atacama y junto con César, abordamos la problemática de la fauna extinta durante la transición Pleistoceno-Holoceno en el centro-norte del país.

Cuando se revisan sus publicaciones se advierte claramente la preocupación en las interacciones entre humanos y sus medioambientes variables, asociados a adaptaciones marítimas. Precisamente, su gran laboratorio lo fue el Norte Semiárido en torno al poblamiento finipleistocénico y los procesos sociales derivados, con énfasis en las relaciones ecológicas desde las micro depresiones costeras a las amplias cuencas interiores. Conversamos largamente sobre las ocupaciones tempranas de TaguaTagua (Cuchipuy) donde logró valorar la información disponible al interior de un programa de cooperación internacional vía CONICYT. Ciertamente, estaba incluido en una red académica española a través de la Universidad de Rovira (Terragona), donde ejerció docencia en su programa de postgrado.

Sin embargo, su mayor energía recayó en la cuenca del Choapa, donde revisó su arte rupestre y no dudó en cómo conservarlo; y por toda esa costa evaluó los recursos marinos y la etnografía de las gentes que aún manejaban relatos de pesca y recolecciones marinas tradicionales. Al tanto, mantenía regularmente las prospecciones sistemáticas en torno a su "lugar" favorito: Los Vilos. Allí comenzó a organizar una fina secuencia cronológica desde los primeros humanos presentes a los 13.000 años AP basada en dataciones radiocarbónicas. Paralelamente definió diferentes patrones de asentamientos en términos de continuidad y discontinuidad, reconociendo los ambientes más estimulantes (v.gr. regímenes hidráulicos).

Que mejor que en esa región privilegiar la cuestión "paleoindio" y la explotación de fauna extinta contemporánea con las ocupaciones humanas en el marco de la evolución y organización del paisaje. Sumó a su espíritu "litiquero", un cuidadoso rastreo de las rocas más eficientes (v.gr. cuarzo y cristal de roca), a través de la ubicación de sus fuentes, para proponer una cadena de talla desde las canteras y talleres hasta la identificación de los instrumentos usados en campamentos concretos (áreas de actividades), estableciendo relaciones entre movilidad y tecnología.

Siguiendo la secuencia se comprometió con los primeros pescadores del Complejo Huentelauquén, evaluando los patrones tecnológicos y los modos de subsistencia y dietas costeras. Pero, además se introdujo en las sociedades formativas al revisar la presencia Molle, las relaciones trasandinas y asentamientos cordilleranos del Chile Central... 
hasta la problemática lítica en espacios mapuches (Isla de Mocha).

Frente a estos temas debidamente publicados, Donald siempre dispuso sus datos en relación a las fuentes de subsistencia y sus escenarios paleoambientales de tiempos finipleistocénicos a holocénicos tempranos y tardíos, tanto en la costa como en el interior inmediato. Es que fue tal su pasión por comprender temas tan diversos entre sí, pero unidos por la construcción de un paisaje común, que logró identificar notables respuestas diferenciadas en el trayecto secuencial. Hasta tocó el tema de las relaciones entre el rol de la infancia y el aprendizaje lítico, que culminó con el registro paradigmático de las puntas Fell y esos esqueletos humanos considerados los más tempranos del Pacífico sudamericano (11.200 años cal AP), asociados por el análisis isotópico con dietas marinas. Eran costeros y con ello Donald terminó por convencerse que "sus" antepasados efectivamente eran antiguos costeros como él... y lo más importante: habían utilizado la vía costera para poblar un espacio antes desocupado. Ese artículo ha logrado un enorme impacto en la opinión científica internacional.

De lo anterior se desprende que, si hay algo que Donald nos enseñó siempre, fue cómo examinar los espacios acotados a discreción, como si realmente fuera el patio de su casa, aplicando un detenido análisis del proceso de formación de cada sitio. De tal modo que, frente a restos de megafauna, algunas veces casi expuestos, debería probar la presencia humana a pesar de las intervenciones de los procesos erosivos y de deflación. En este sentido la evaluación crítica del efecto palimpsesto le permitió esclarecer cuando un yacimiento era efectivamente una respuesta humana. Esto explica que se trasformara en un experto en los procesos de diagénesis esqueletarios y así dilucidar los eventos de formación y trasformación de sitios. Buscaba esclarecer las diferencias entre las intervenciones naturales de las culturales en esos sitios tempranos donde Donald solía decir que toda excavación aborda complejidades inesperadas.

A la par de detectar sitios finiplesitocénos con un seguimiento del manejo de sus recursos y tafonomía lítica, estableció vínculos entre canteras, talleres y artefactos in situ, en un meditado análisis de las cadenas operativas de talla tanto en evidencias de superficie como en leves o profundos depósitos estratificados. En verdad, al interior de todos los territorios articulados, se levantó su pasión "litiquera" trasfiriendo a sus alumnas y alumnos su mayor divertimento y capital empírico desde sus propias manos. Era muy visible la falta de esa falange mítica, porque jamás se supo, ni los más cercanos, cual fue la verdadera causa de su desaparición...

Ocurrió entonces que este "Profe Flaquín", el gran "picapiedrero", para sus alumnos y colegas, fue transformándose en un maestro formador de cuadros científicos que siguieron sus enseñanzas a plenitud, desde el faenamiento de un lobo de mar, hasta sus interminables enseñanzas sobre cómo identificar los patrones de uso de los artefactos líticos.

Todo esto estuvo matizado entre pisos excavados en bordes de depósitos paleolacustres o principalmente en los numerosos conchales, exponiendo depósitos rigurosamente decapados con sus artefactos, residuos y presas in situ. Se destaca su franca y correcta obsesión por la tafonomía, al hacer "hablar" a las conchas, estableciendo no sólo su información malacológica, sino también determinar las variaciones paleoambientales a partir de moluscos y crustáceos. Fue así que se trasformó en un coleccionista de moluscos terrestres dulceacuícolas clasificados sistemáticamente con su hermano Douglas, con muestras obtenidas a lo largo del país. Donde estuvieran los "prontuariaba" vivos o muertos... Los análisis de polen, como marcadores de cambios climáticos, junto con Antonio Maldonado, siempre fue una crucial línea de información. Como si fuera poco, hasta se preocupó del patrimonio cultural arqueológico en ciertas áreas de protección silvestre. Entre tantas acciones referidas, no sólo lo absorbió al Semiárido sino que aun abarcó otros problemas regionales. Se acercó a temas como el paleoindio del extremo sur por las tierras de los Selk`nam, observando la materialidad lítica en Tierra del Fuego hasta auscultar ese "fondo de saco" para fines comparativos. De paso, estudió objetos arqueológicos históricos en Magallanes.

Fue invitado por su experticia lítica y estrategias interdisciplinarias a abordar problemas del territorio árido. Se recuerda sus estudios en el campamento formativo Chiu Chiu 200 del Loa medio y otro arcaico cercano en Confluencia 2. Colaboró además en los estudios del temprano yacimiento de óxido de fierro de los cerros de Taltal asociado al equipo de Diego Salazar. También aplicó su experiencia en la lítica de la sierra ariqueña, en el sitio Hakenasa, y más recientemente se le invitó a evaluar sitios de la transición Pleistoceno-Holoceno en las tierras bajas del desierto absoluto de Atacama, en alianza con su líder Calogero Santoro, en lo que fue su última labor como coinvestigador de un proyecto Fondecyt. Allí se involucró 
como coautor a través de un modelo de exploración predictivo con una base interdisciplinaria donde Donald aplicó su larga experiencia y talento crítico arqueológico.

A juzgar por sus avances en las investigaciones en el centro del país, fue también invitado a participar en diversos colectivos internacionales donde aplicó los análisis líticos de micro huellas y patrones de desgastes. Paralelamente, contribuyó a evaluar el rol de los análisis geológicos y geomorfológicos, principalmente de los movimientos tectónicos y niveles variables de aterrazamientos en la costa ecuatoriana. Asimismo, analizó los artefactos líticos de Tula en México, incluyó un estudio crítico sobre las industrias del Complejo Andino de Buriles y participó en trabajos en torno a los homínidos del famoso yacimiento español de Atapuerca, invitado por el Instituto Catalán de paleontología humana y evolución social.

Claramente, ya entrado en su creación científica madura, Donald planteó un análisis crítico del estado actual de la arqueología chilena, mientras que mantenía su apertura hacia cuestiones mayores a nivel latinoamericano amparado en los principios observados en los talleres de arqueología social acogidos en sus reuniones de México.

Esa mezcla curiosa entre su ADN inglés-italiano-nativo americano y su crianza costera dieron cuenta de un singular imaginario donde la metódica disciplina se entrecruzaba con actos "rituales" motivados por quien sabe qué extraños "ancestros" locales. Sólo él podía ejecutar actos algo surrealistas consistentes en bañarse desnudo con envolturas de algas que le evocaban sospechosas ceremonias del pasado... Ciertamente, nadie negaría que sus terrenos fueran una adecuada mezcla de rigor científico-docente, pero muy entretenido a la vez por esta singular mezcla genética y cultural. Yo sólo puedo probar que en nuestras conversaciones compartimos esa vieja virtud de tragarnos la noche sin dejar ni la más mínima huella de su oscuridad.

Al final de su vida académica plena nos reunimos para programar una acción editorial conjunta. Acordamos juntar nuestros datos y publicar un libro sobre Quereo. Alcanzamos a pensar sobre el montaje y aun su índice hasta que suspendimos los encuentros a raíz de los inicios de sus malestares. Otra obra inconclusa se paralizó en esos tiempos, cuando junto a César Méndez y Calogero Santoro, comenzamos en varias reuniones en su laboratorio a proponer a varios autores para un libro sobre los primeros poblamientos en Chile a cargo de la Editorial Universita- ria. Logramos enviar las primeras circulares y siguiendo su entusiasmo, lo haremos con más energía para dedicárselo en su memoria. Entre medio quedaron varios manuscritos en revisión y por enviar a revistas científicas, una prueba más de que Donald se aferró a la vida hasta la paz final.

Entre sus reconocimientos a Julio Montané y Felipe Bate, por compartir sus afanes en los primeros poblamientos compartidos en México, sentía profundamente la herencia geoarqueológica que nos dejó nuestro querido geólogo Juan Varela (1938-1993). Esa orientación la compartimos a tal punto que ambos mantuvimos una franca admiración por Juan. De este modo, Donald advirtió que tanto sus investigaciones, como las de otros colegas, habían enfatizado una visión interdisciplinaria que ameritaba una reunión particular. Comenzó a planear la organización del "II Taller Latinoamericano de Geoarqueología". Su último proyecto FONDECYT "Primer poblamiento en el Semiárido de Chile. Interacción entre seres humanos y fauna extinta en Los Vilos", lo había motivado para sintetizar en este evento internacional sus resultados junto a otras ponencias de áreas vecinas. Quería organizarlo en Los Vilos como un conversatorio bajo un espíritu colectivo, junto a sitios concretos. Con César Méndez estaban preparados para con ducir este Seminario con alta convocatoria hasta que sus severas molestias lo alejaron de su propio escenario. Sin embargo, fue gracias a Roxana Seguel, llave maestra de su equipo y de su vida, quien lo organizó en su totalidad.

Pese a sus padecimientos, pero firme como un paleoislote o un "Don Roble" en las palabras de Santoro, Donald tuvo el coraje de terminar su tesis doctoral conducida por Gustavo Politis, enfocada a "El primer poblamiento de la costa Pacífica sudamericana: el complejo Huentelauquén". No alcanzó a defenderla. No obstante, para nosotros, desde su ausencia Donald será siempre el Maestro por excelencia, un brillante Profesor Doctor por su propia magnificencia. Inmediatamente antes de su ausencia definitiva, en absoluta pasividad, me llamó para preguntarme por mi salud, y de paso nos invitó a su casa, junto a Calogero, siempre y cuando llegáramos con su whisky favorito. Qué duda cabe, lo beberemos para nuestro próximo encuentro, acompañados de Douglas, esta vez buceando estrellas en el fondo del universo oceánico de Los Vilos.

San Pedro de Atacama 2 de noviembre 2015 